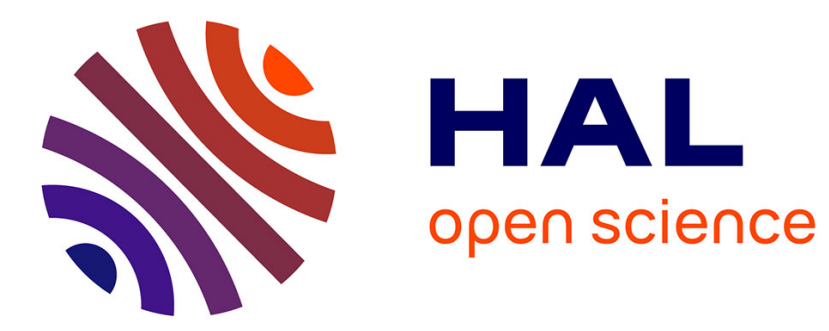

\title{
Deuterium and oxygen 18 in precipitation: Isotopic model, including mixed cloud processes \\ Philippe Ciais, Jean Jouzel
}

\section{To cite this version:}

Philippe Ciais, Jean Jouzel. Deuterium and oxygen 18 in precipitation: Isotopic model, including mixed cloud processes. Journal of Geophysical Research, 1994, 99 (D8), pp.16.793-16.803. 10.1029/94JD00412 . hal-02923778

\section{HAL Id: hal-02923778 \\ https://hal.science/hal-02923778}

Submitted on 3 Feb 2021

HAL is a multi-disciplinary open access archive for the deposit and dissemination of scientific research documents, whether they are published or not. The documents may come from teaching and research institutions in France or abroad, or from public or private research centers.
L'archive ouverte pluridisciplinaire HAL, est destinée au dépôt et à la diffusion de documents scientifiques de niveau recherche, publiés ou non, émanant des établissements d'enseignement et de recherche français ou étrangers, des laboratoires publics ou privés. 


\title{
Deuterium and oxygen 18 in precipitation: Isotopic model, including mixed cloud processes
}

\author{
Philippe Ciais ${ }^{1}$ and Jean Jouzel ${ }^{2}$ \\ Laboratoire de Modelisation du Climat et de l'Environnement, DSM, Gif-sur-Yvette, France
}

\begin{abstract}
Modeling the isotopic ratios of precipitation in cold regions meets the problem of "switching" from the vapor-liquid transition to the vapor-ice transition at the onset of snow formation. The one-dimensional model (mixed cloud isotopic model (MCIM)) described in this paper focuses on the fractionation of water isotopes in mixed clouds, where both liquid droplets and ice crystals can coexist for a given range of temperatures. This feature is linked to the existence of specific saturation conditions within the cloud, allowing droplets to evaporate while the water vapor condensates onto ice crystals. The isotopic composition of the different airborne phases and the precipitation is calculated throughout the condensation history of an isolated air mass moving over the Antarctic ice sheet. The results of the MCIM are compared to surface snow data both for the isotopic ratios and the deuterium excesses. The sensitivity of the model is compared to previous one-dimensional models. Our main result is that accounting specifically for the microphysics of mixed stratiform clouds (BergeronFindesein process) does not invalidate the results of earlier modeling studies.
\end{abstract}

\section{Introduction}

The study of the deuterium and oxygen 18 distributions in natural waters proves to be very useful in such fields as cloud physics, climatology, hydrology, and paleoclimate studies. Usually, data acquisition is combined with the modeling of isotopic fractionations occurring at various stages of the water cycle and the interpretation takes advantage of the relationships which exist between isotopic distributions and various, generally climate-related parameters. The purpose of isotopic models is to predict the global scale deuterium and oxygen 18 distributions in water vapor, clouds, and precipitations. For both isotopes, the isotopic mass ratios $(R)$ are conventionally expressed in the $\delta$ scale relative to the $S M O W$ standard:

$$
\delta=\frac{R-R_{\text {SMOW }}}{R_{\text {SMOW }}} \times 1000 \text { (units \%o) }
$$

Isotopic models fall in two categories. Dynamically simple models deal with processes occurring in isolated air masses. They are generally based on Rayleigh condensation models assuming immediate removal of precipitation [Dansgaard, 1964; Friedman et al., 1964; Taylor, 1972; Siegenthaler and Matter, 1983; Jouzel and Merlivat, 1984]. Though being indispensable tools in understanding field isotope data and their relationships with climatic variables, these simple models do not account for the complexity of the dynamical processes involved in the formation of precipitation. To deal with these latter aspects, the isotopic cycles have been

\footnotetext{
${ }^{1}$ Also at Stable Isotope Laboratory, University of Colorado INSTAAR, Boulder.

${ }^{2}$ Also at Laboratoire de Glaciologie et de Géophysique de l'Environnement, St. Martin-d'Hères, France.

Copyright 1994 by the American Geophysical Union.

Paper number 94JD00412.

0148-0227/94/94JD-00412\$05.00
}

introduced into general circulation models (GCMs), that consider this dynamical complexity [Joussaume et al., 1984; Jouzel et al., 1987]. Besides those two approaches, an intermediate two-dimensional isotopic model has been developed by Fisher [Fisher and Alt, 1985; Fisher, 1990]. Both simple models and GCMs are very complementary and are now being developed in parallel.

One of the advantages of simple models is their ability to account for the complexity of cloud microphysics and its interplay with the various fractionation processes occurring at each phase change of the water in the atmosphere, while those processes can only be parameterized in dynamically complex models. As reviewed by Jouzel [1986], this complexity is due to the following reasons: Two types of isotopic fractionations are involved. An equilibrium fractionation, arising from lower saturation vapor pressure of heavy species $\mathrm{HDO}$ and $\mathrm{H}_{2}{ }^{18} \mathrm{O}$ compared to the main component $\mathrm{H}_{2}{ }^{16} \mathrm{O}$, and a kinetic effect resulting from differences in the molecular diffusivities in air of these molecules. Liquid and solid phases have to be treated separately, because, unlike liquid droplets, the cloud solid (ice crystals, large ice particles, or hailstones) is not isotopically homogeneous. Ice crystals are out of isotopic equilibrium due to low diffusion of molecules in solid and the variable history of the air mass producing the solid. Liquid droplets whose radius is less than $30 \mu \mathrm{m}$ can be considered at any time in isotopic equilibrium with the surrounding water vapor [Jouzel et al., 1975]. Raindrops of radius larger than $30 \mu \mathrm{m}$ are out of isotopic equilibrium because of their large isotopic relaxation times.

With this in mind, Jouzel et al. [1980] and Federer et al. [1982] have incorporated into a cloud model the equations describing the isotopic fluxes among vapor, droplets (cloud water), raindrops (rainwater), ice crystals (cloud ice), graupels, and hailstones. This model represents a very elaborate description of isotopic processes occurring in clouds, but its use is limited to the study of isolated convective clouds and 
it would not be appropriate for describing the isotopic history of an air mass from its oceanic source to, for example, the polar ice caps. Also, it does not adequately account for the two important following points: First, one has to consider a drop size spectrum rather than a fixed radius drop population, as partially addressed by Federer et al. [1982]. However, this point is important only for convective clouds and will not be further discussed here. Second, if both liquid droplets and ice crystals are to coexist for a given range of temperatures, then neither the droplets nor the crystals will be in isotopic equilibrium [Jouzel and Merlivat, 1984].

This is due to the so-called Bergeron-Findesein process which plays a key role in the formation of ice crystals [Rogers, 1979]. This physical mechanism discussed by Bergeron [1935] had been formulated as early as 1911 by Wegener (quoted by Mason [1971]): "The vapor tension will adjust itself to a value in between the saturation values over ice and water. The effect of this must then be that condensation will take place continually until the liquid phase is entirely consumed." In mixed clouds, i.e., clouds containing both liquid water and ice, the environment should thus be undersaturated with respect to the liquid phase while supersaturated with respect to the solid phase. If so, the liquid droplets tend to evaporate, while two phase transitions can lead to ice formation: direct deposition of vapor onto the existing crystals by inverse sublimation and freezing of supercooled droplets. Ice crystals usually begin to appear in clouds when the temperature drops below $-15^{\circ} \mathrm{C}$, while supercooled droplets end at temperatures as low as $-40^{\circ} \mathrm{C}$, the homogeneous freezing threshold [Rogers, 1979].

From an isotopic point of view, the Bergeron-Findesein process may be neglected in convective clouds, as noted by Federer et al. [1982], but probably not in stratiform clouds where the formation of polar precipitation takes place [Jouzel and Merlivat, 1984]. The fact that the vapor pressure is likely above the saturation pressure over ice had already been accounted for by Jouzel and Merlivat [1984] through the formulation of a kinetic fractionation during ice formation. We will adopt their description of the isotopic effects at the vapor-ice phase transition. Besides, during the BergeronFindesein process in mixed clouds the vapor pressure is also below the saturation pressure over liquid water. Therefore droplets will not be in isotopic equilibrium with respect to the vapor, which requires to account for a kinetic fractionation associated to the vapor-liquid transition.

Hereinafter, we present an isotopic cloud model which fully includes such nonequilibrium processes. This so-called mixed cloud isotopic model (MCIM) is directly in the line of those developed by Jouzel and Merlivat [1984] for the solid phase (referred hereinafter as RMK model) and by Merlivat and Jouzel [1979] for the liquid phase. These models are now currently used in interpreting the isotopic content of polar snow concerning the isotope-temperature relationship. Combined with these two models, the MCIM will provide a full description of isotopic processes occurring in an air mass from its oceanic origin up to the cold ice sheet regions. The deuterium excess (a linear combination of both isotopes defined as $d=\delta D-8 \delta^{18} \mathrm{O}$ ) will also be examined with the MCIM. The study of $d$ makes it possible to infer climatic information about the conditions of temperature and humidity prevailing at the evaporative ocean source of the vapor [Jouzel et al., 1982; Johnsen et al., 1989; Dansgaard et al.,
1989; Petit et al., 1991; Fisher, 1991]. Kinetic fractionation effects are slightly greater in proportion for oxygen 18 than for deuterium. As $d$ couples both isotopes, it is a very relevant parameter to examine in the MCIM which deals specifically with kinetic fractionations. We will explore the properties of $d$ in the MCIM as in the work of Petit et al. [1991].

\section{Description of the Model}

Vapor, Liquid, and Ice in the Mixed Cloud Isotopic Model (MCIM)

The variable $T$ refers to the cloud temperature (or condensation temperature), the natural working variable of onedimensional isotopic models. For model-data comparison we converted the observations from ground to cloud temperature using the empirical linear function derived by Jouzel and Merlivat [1984] over the Antarctic ice sheet $\left(T_{\text {cloud }}=\right.$ $0.67 \times T_{\text {ground }}-1.2$ ). Figure 1 describes the condensation history of an air mass according to three hypotheses. Figure la is a Rayleigh distillation for which the condensed phase is removed as soon as it forms in the cloud. Figure $1 \mathrm{~b}$ is the model of Jouzel and Merlivat [1984] (RMK model) where some liquid remains in the cloud above $T_{s}$. Figure $1 \mathrm{c}$ is the MCIM model of this paper. From the ocean evaporation to the apparition of the first ice crystal at $T_{s}$ the MCIM is strictly identical to the model of Jouzel and Merlivat. Specifically, the moist air is lifted up till its dew point is reached, then starts the condensation process; a certain amount of liquid is kept into the cloud and the vapor is saturated with the liquid (the liquid is in isotopic equilibrium). In the interval $\left(T_{s}, T_{w}\right)$ the Bergeron-Findesein process occurs. Ice appears in the system at $T_{s}$, but in contrast to the RMK model, supercooled droplets are still present down to $T_{w}$. Below $T_{w}$ the liquid has entirely disappeared and the cloud is only composed of ice and vapor. At that point, due to successive removal by precipitation, the amount of airborne ice in the cloud is very low. The system is then fairly close to the Rayleigh distillation conditions.

\section{Supersaturation Function}

The water vapor partial pressure in the cloud $\left(e_{w}\right)$ drives the amount of the different airborne phases. According to the Bergeron-Findesein hypothesis, the value of $e_{w}$ lies between the saturation over liquid water $\left(e_{l}^{s}\right)$ and the saturation over ice $\left(e_{i}^{s}\right)$. This is shown in Figure 2. Instead of the water vapor partial pressures, we prefer to speak in terms of supersaturation, a commonly used variable in this type of studies. The supersaturation of the vapor with respect to the ice $\left(S_{i}\right)$ is defined as

$$
S_{i}=\frac{e_{w}}{e_{i}^{s}}
$$

There are only very few measurements of $S_{i}$ in polar clouds [Miller and Schverdtferger, 1972; Miller, 1973]. The exact temperature dependence of $S_{i}$ over the Antarctic cannot be constrained by observations. Therefore the function $S_{i}(T)$ was prescribed in the model for three separate temperature domains. For $T>T_{s}$, we assume that the vapor is exactly saturated with respect to the liquid droplets in the cloud. This is expressed by 


$$
S_{i}=\frac{e_{l}^{s}}{e_{i}^{s}}
$$

For $T<T_{w}$, dealing with the vapor-ice transition only, $S_{i}$ is taken to be a linear function of the cloud temperature. This choice is somewhat arbitrary but has proven robust enough to describe correctly the variability of the observed $\delta$ in snow, including the behavior of the deuterium excess
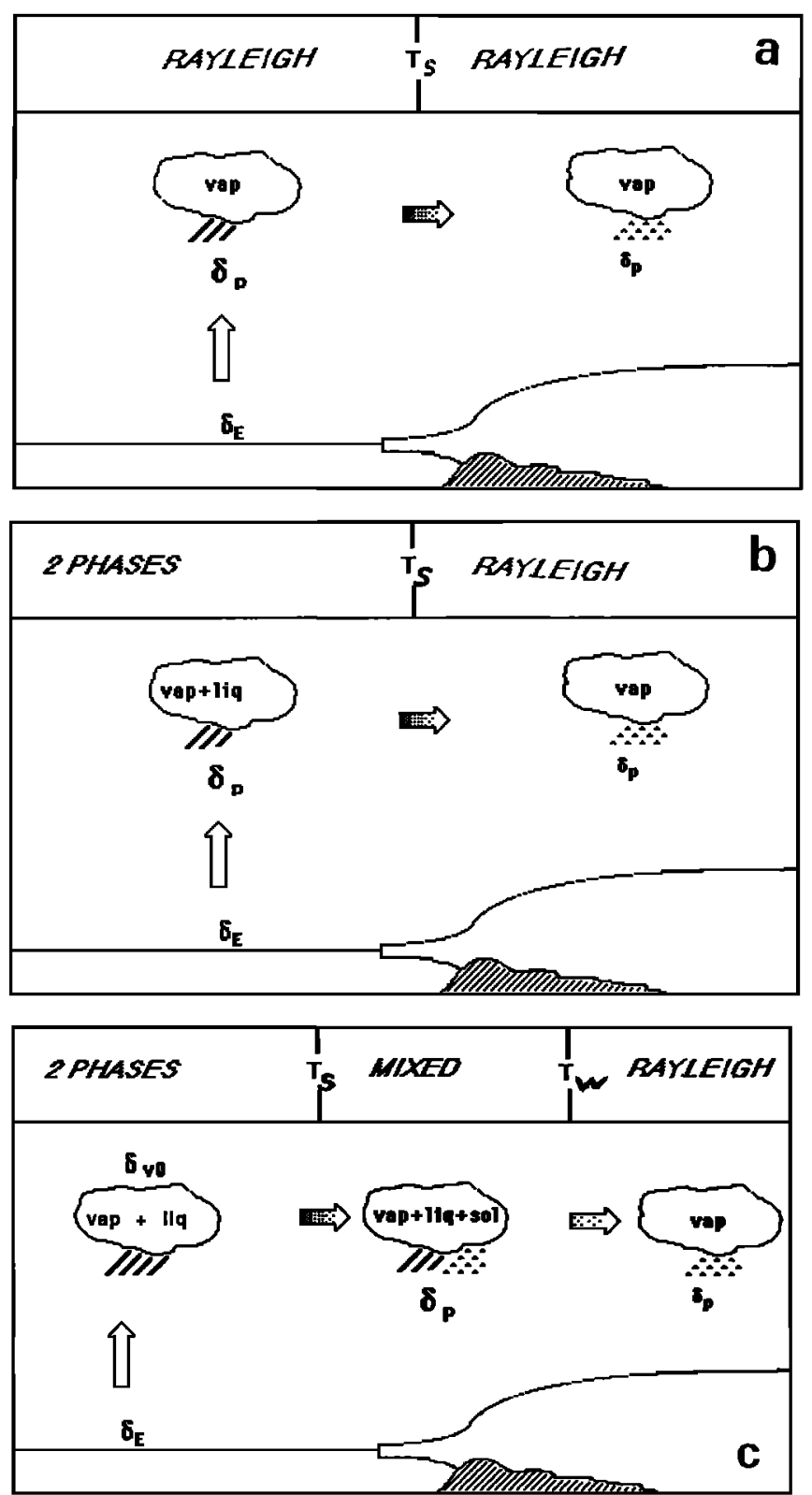

Figure 1. Three generations of one-dimensional isotopic models: (a) classical Rayleigh distillation with a switch between formation of liquid and formation of snow at $T=$ $T_{s}$. (b) RMK model of Jouzel and Merlivat [1984]. Above $T_{s}$, some liquid is kept in the eloud, but below $T_{s}$ the model is a Rayleigh distillation. (c) MCIM model. Above $T=T_{s}$, the model is identical to the RMK. Between $T=T_{s}$ and $T=$ $T_{w}$, both ice crystals and supercooled droplets coexist in the system, according to the Bergeron-Findesein process. Below $T=T_{w}$, there is no more liquid in the cloud and the model approaches a Rayleigh distillation with ice and vapor.

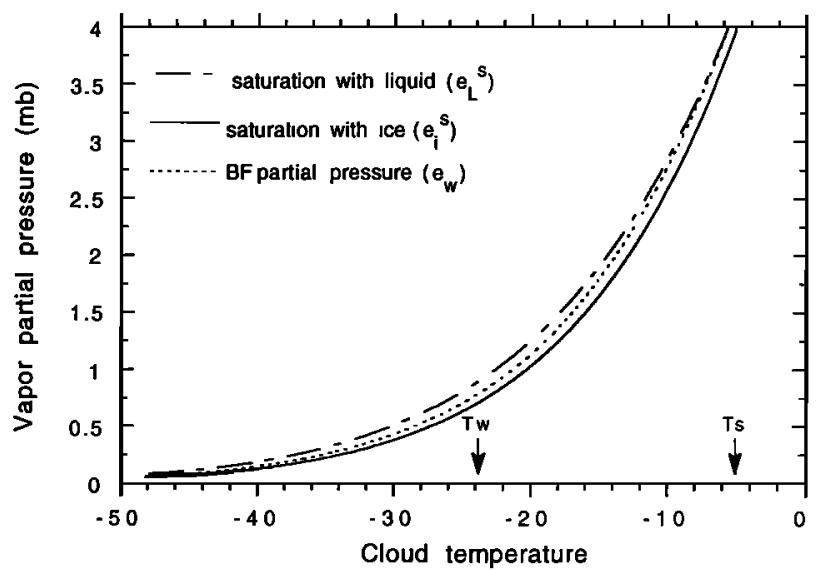

Figure 2. Short-dashed curve, partial pressure of water vapor in the MCIM model as a function of cloud temperature. The partial pressure plotted here corresponds to a supersaturation of $S_{i}=1.02-0.0038 \times T$ below $T=T_{w}$. In between $\left(T_{s}, T_{w}\right)$ the partial pressure obeys the Bergeron-Findesein hypothesis. Solid curve, saturation pressure for the vapor-ice transition. Long-dashed curve, saturation pressure for the vapor-liquid transition.

[Johnsen et al., 1989; Fisher, 1991; Petit et al., 1991]. In the standard run of the MCIM model, $S_{i}$ corresponds to the "preferred case" of Petit et al. [1991].

$$
\begin{gathered}
S_{i}=p+q \times T \\
p=1.02, \quad q=-0.0038
\end{gathered}
$$

In a recent paper, Fisher [1991] outlined that the function $S_{i}$ probably takes very different values from one storm to the other, or even within the same cloud. Expression (3) should be seen as an average supersaturation relative to several precipitation events, from which we simulate the mean values of $\delta$ and $d$ in snow. Finally, in the interval $\left(T_{s}, T_{w}\right)$ which defines the limits of the Bergeron-Findesein process, we impose continuity both for $S_{i}$ and its derivative. Gradually, $S_{i}$ becomes lower than the curve describing the saturation over liquid water at $T_{s}$ and reaches the curve (equation (3)) of supersaturation over ice at $T_{w}$. Figure 3 shows the shape of the $S_{i}$ function of temperature. One cannot entirely dismiss the possibility that as far as liquid water is available to evaporate, the vapor pressure is maintained exactly at its saturation value over the liquid. Such a possibility can be accounted for by adjusting the value of $p$ and $q$ (Figure 3). In any case, the discontinuity in the supersaturation function that occurred in previous works when switching from equation (1) to equation (3) at the onset of the formation of snow does not exist any longer. One of the principal advantages of the MCIM is to fill the modeling gap of intermediate temperatures $\left(T_{s}, T_{w}\right)$ by accounting for mixed cloud processes.

\section{Cloud Model}

In this section we describe how the mass transfers among vapor, liquid water, and ice are formulated in the MCIM for the Bergeron-Findesein process. Figure 4 summarizes the microphysical mechanisms interplaying among the different phases. The liquid droplets disappear because of evaporation and freezing, whereas the vapor condenses into ice. At 


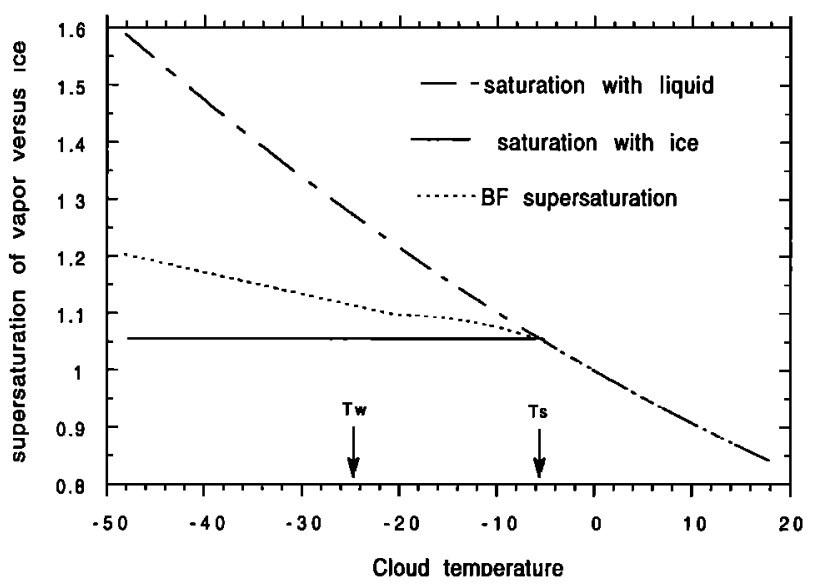

Figure 3. Supersaturation function $\left(S_{i}\right)$ of the vapor with respect to cloud ice in the MCIM model. Vertical arrows are the boundaries of the Bergeron-Findesein domain $\left(T_{s}, T_{w}\right)$. Solid curve, $S_{i}$ for the vapor-ice transition at equilibrium. Long-dashed curve, $S_{i}$ for the liquid-vapor transition at equilibrium. Short-dashed curve, intermediate $S_{i}$, characteristic of the Bergeron-Findesein process. Such an intermediate $S_{i}$ function is assumed to be linear below $T=T_{w}\left(S_{i}=\right.$ $1.02-0.0038 \times T)$.

each step, the precipitation processes remove a fraction of the cloud liquid water and of the cloud ice. We assume that the condensation history of the moist air mass follows three assumptions: (1) no mixing with the outside air, (2) no large raindrops, (3) no isotopic fractionation when freezing a droplet into an ice particle (the freezing characteristic time of droplets must be short compared to their evaporative characteristic time). The mixing ratios of the vapor, the cloud liquid droplets, and the cloud ice are denoted as $m_{v}, m_{l}$, and $m_{i}$. The removal of liquid (ice) by precipitation is noted $f_{l}\left(f_{i}\right)$, the evaporative flux of droplets $\boldsymbol{P}_{l v}$, the freezing of liquid droplets $\boldsymbol{P}_{l i}$, the condensation of vapor into ice $\boldsymbol{P}_{v i}$. Note that the term "liquid precipitation" refers only to the fact that they are derived from the cloud liquid. In a real cloud, especially above an ice sheet, droplets falling to the surface are likely to collide on ice crystals and freeze immediately, hence reaching the ground under solid state [Pruppacher and Klett, 1978; Rogers, 1979; Johnsen et al., 1989]. This freezing nevertheless will not affect their isotopic ratio, as long as the surface temperature is low enough to prevent secondary evaporation during the fall of the droplets. The mass balance of the system is expressed by

$$
d m_{v}+d m_{l}+d m_{i}+f_{l}+f_{i}=0
$$

Water vapor. The mixing ratio of the vapor $\left(m_{v}\right)$ is controlled by the air pressure $(P)$ and the water vapor partial pressure $\left(e_{w}\right)$ which is a function of temperature. This is expressed by

$$
m_{v}=\frac{18 \times e_{w}}{29 \times P-18 \times e_{w}}
$$

Liquid water. The liquid mixing ratio $\left(m_{l}\right)$ is controlled by the evaporation, the freezing and the precipitation of the cloud droplets. The formulation of $\boldsymbol{P}_{l v}$ and $\boldsymbol{P}_{l i}$ is detailed below. The liquid precipitation $\left(f_{l}\right)$ is adjusted in such a manner that $m_{l}$ decreases continuously from $m_{l}=m_{10}$ at $T$
$=T_{s}$ ( $m_{10}$ is the amount of liquid present in the cloud when the Bergeron-Findesein process begins) to $m_{l}=0$ at $T=$ $T_{w}$. For each condensation step, the variation in the liquid mixing ratio is expressed by

$$
d m_{l}=-P_{l v}-P_{l i}-f_{l}
$$

Ice crystals. Ice is formed in the cloud by the freezing of liquid droplets and by the condensation of vapor. It is removed according to the precipitation flux $\left(f_{i}\right)$. The evolution of the ice mixing ratio $\left(m_{i}\right)$ obeys to

$$
d m_{i}=P_{v i}+P_{l i}-f_{i}
$$

The solid precipitation $\left(f_{i}\right)$ is left adjustable in our model, which serves to control the amount of cloud ice. Specifically, we used

$$
\begin{array}{cc}
f_{i}=C_{1 \mathrm{prec}}\left(m_{i}\right) & \text { if } f_{i}<d m_{i} \\
f_{i}=C_{2 \operatorname{prec}}\left(d m_{i}\right) & \text { if } f_{i} \geq d m_{i}
\end{array}
$$

The global sensitivity of the model to $f_{i}$ is examined below. The formulation of equations (8) and (9) has no physical basis. Both precipitation coefficients $C_{1 \text { prec }}$ and $C_{2}$ prec are just a convenient way to fix the amount of cloud ice in a plausible manner (see Figure 5). Typically, the precipitation

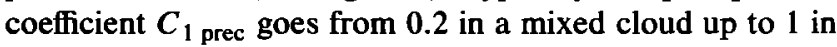
a Rayleigh distillation. The precipitation coefficient $C_{2}$ prec is chosen at around 1.01 in a standard run of the MCIM. For the mass balance equation of the system to be entirely determined, we now have to provide an estimate for the fluxes describing the transfers that occur among the three phases.

Evaporation of liquid water. Cloud droplets evaporate because of the undersaturated conditions during the Bergeron-Findesein process. The evaporation flux $\left(P_{l v}\right)$ is assumed to be proportional, first to the departure from saturation over liquid water and second to the liquid mixing ratio. As a consequence the more the distance from the saturation increases, the greater the evaporative flux will be. This is

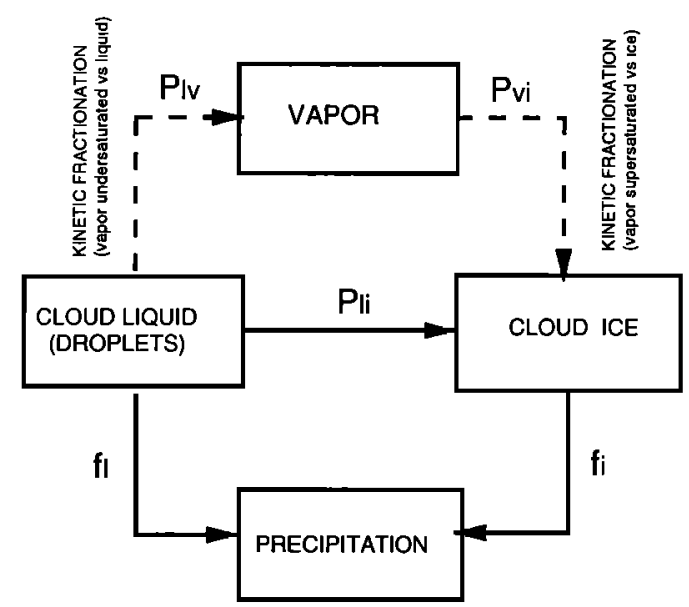

Figure 4. Mass transfers between the different constituents in the cloud. Dashed lines point out fluxes which are associated with an isotopic fractionation. Such fluxes involve mass transfer due to diffusion processes. The precipitation (removed from the cloud) and the freezing of liquid droplets are rapid processes and they do not yield any isotopic fractionation. 
expressed by equation (10) below, in which the evaporation coefficient $C_{e}$ is left adjustable. In the standard run, $C_{e}$ is set to $10^{-4}$.

$$
P_{l v}=C_{e}\left(1-S_{l}\right) m_{l}
$$

$S_{l}$, supersaturation of the vapor with respect to the cloud liquid, is defined by

$$
S_{l}=\frac{e_{w}}{e_{l}^{s}}
$$

Freezing of liquid water. The freezing of a fraction of the droplets occurs as the temperature decreases [Bigg, 1953]. The corresponding flux $\left(P_{l i}\right)$ relates to the liquid mixing ratio according to equation (12). The freezing coefficient $C_{f}$ has to be adjusted by trial and error. In the standard run of the model, we used $C_{f}=10^{-4}$. The isotopic model is not very sensitive to the value of $C_{f}$ when $C_{f}$ varies between 0 and $\approx 10^{-3}$.

$$
P_{l i}=C_{f}\left(m_{l}\right)
$$

Condensation of vapor. The condensation of vapor molecules on ice crystals $\left(P_{v i}\right)$ is deduced from the decrease in the vapor mixing ratio at each condensation. This is expressed by

$$
P_{v i}=-\frac{\partial m_{v}}{\partial T} d T
$$

\section{Isotopic Cloud Model}

We now examine the isotopic implications of equations (4) to (13). The Bergeron-Findesein process consists of two phase transitions, both out of equilibrium (evaporation of droplets, condensation of vapor into ice). The isotopic effects associated with each transition are different, although they all yield an isotopic depletion of the vapor.

Isotopic fractionation during the Bergeron-Findesein process. For the vapor-ice transition in an environment where the vapor is supersaturated over ice, an extra kinetic effect is superimposed to the classical equilibrium fractionation coefficient $\alpha_{i}$ [Jouzel and Merlivat, 1984]. We used their formulation of the total fractionation coefficient $\left(\alpha_{k i}\right)$.

$$
\alpha_{k i}=\alpha_{i} \alpha_{k} \text { with } \alpha_{k}=\frac{S_{i}}{\alpha_{i}\left(D / D^{\prime}\right)\left(S_{i}-1\right)+1}
$$

The value of $\alpha_{k}$ depends on the supersaturation function $S_{i}(T)$. In a recent work, Fisher [1991] improved the formulation of $\alpha_{k}$ by considering the dependence of all the variables on the ambient air temperature. This improvement produces virtually no changes in the model results for either isotope and only a small change in the predicted deuterium excess. Hence for the sake of comparison with previous studies, we chose to keep the original formulation of Jouzel and Merlivat [1984] in the MCIM. Using the convention $R_{\text {SMOW }}=1$, we expressed the condensation of isotopic species in the vapor onto ice crystals:

$$
P_{v i}^{\prime}=\alpha_{k i}\left(1+\delta_{v}\right) P_{v i}
$$

As the liquid-vapor transition occurs when the vapor is undersaturated with respect to the liquid, the pertaining fractionation coefficient also includes additional kinetic ef-

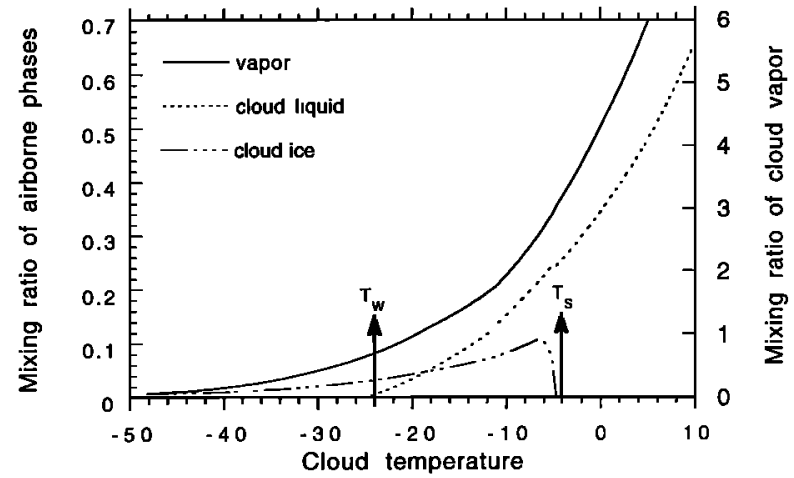

Figure 5. Mixing ratios of the different constituents in the cloud for the standard run of the MCIM model. Vertical arrows mark the boundaries of the Bergeron-Findesein domain $\left(T_{s}, T_{w}\right)$. The cloud already contains some liquid at high temperatures $\left(T>T_{s}\right)$. Ice starts forming at $T=T_{s}$, but it is subsequently removed by precipitation. Liquid droplets disappear completely at $T=T_{w}$ as the result of successive freezing and evaporation.

fects. As far as the liquid phase of the system is composed of small droplets with a mean radius that does not exceed 30 $\mu \mathrm{m}$ [Jouzel et al., 1975], the liquid and vapor phases may be considered to be in pseudoequilibrium and it suffices to replace the equilibrium fractionation coefficient $\left(\alpha_{l}\right)$ by its pseudoequilibrium value $\left(\alpha_{k l}\right)$. The general derivation of $\alpha_{k l}$ for a droplet either growing in a supersaturated environment or evaporating in an undersaturated environment (our case of interest) is detailed in the Appendix:

$$
\begin{aligned}
\alpha_{k l}=S_{l}\left(D^{\prime} f^{\prime}\right)\left[S _ { l } \left(\frac{D^{\prime} f^{\prime}}{\alpha_{l}}-\frac{A D^{\prime} f^{\prime}}{\alpha_{l}}\right.\right. & +A D f) \\
& \left.+\frac{A D^{\prime} f^{\prime}}{\alpha_{l}}-A D f\right]^{-1}
\end{aligned}
$$

Using (16), the liquid isotopic composition is given by

$$
\left(1+\delta_{l}\right)=\alpha_{k l}\left(1+\delta_{v}\right)
$$

Finally, no isotopic change is associated with the freezing of liquid because the freezing is rapid (the time needed to freeze a droplet is much lower than its $e$-folding isotopic relaxation time). This is expressed by

$$
P_{l i}^{\prime}=\left(1+\delta_{l}\right) P_{l i}
$$

Complementary equations (19) to (23) consist of those directly derived from the definition of isotopic ratios. Equation (24) describes the mass balance of isotopic species. Equations (25) and (26) for isotopes are related to (6) and (7) for the dominant species.

$$
\begin{aligned}
m_{v}^{\prime} & =\left(1+\delta_{v}\right) m_{v} \\
m_{l}^{\prime} & =\left(1+\delta_{l}\right) m_{l} \\
m_{i}^{\prime} & =\left(1+\delta_{i}\right) m_{i} \\
f_{i}^{\prime} & =\left(1+\delta_{l}\right) f_{l} \\
f_{i}^{\prime} & =\left(1+\delta_{i}\right) f_{i}
\end{aligned}
$$



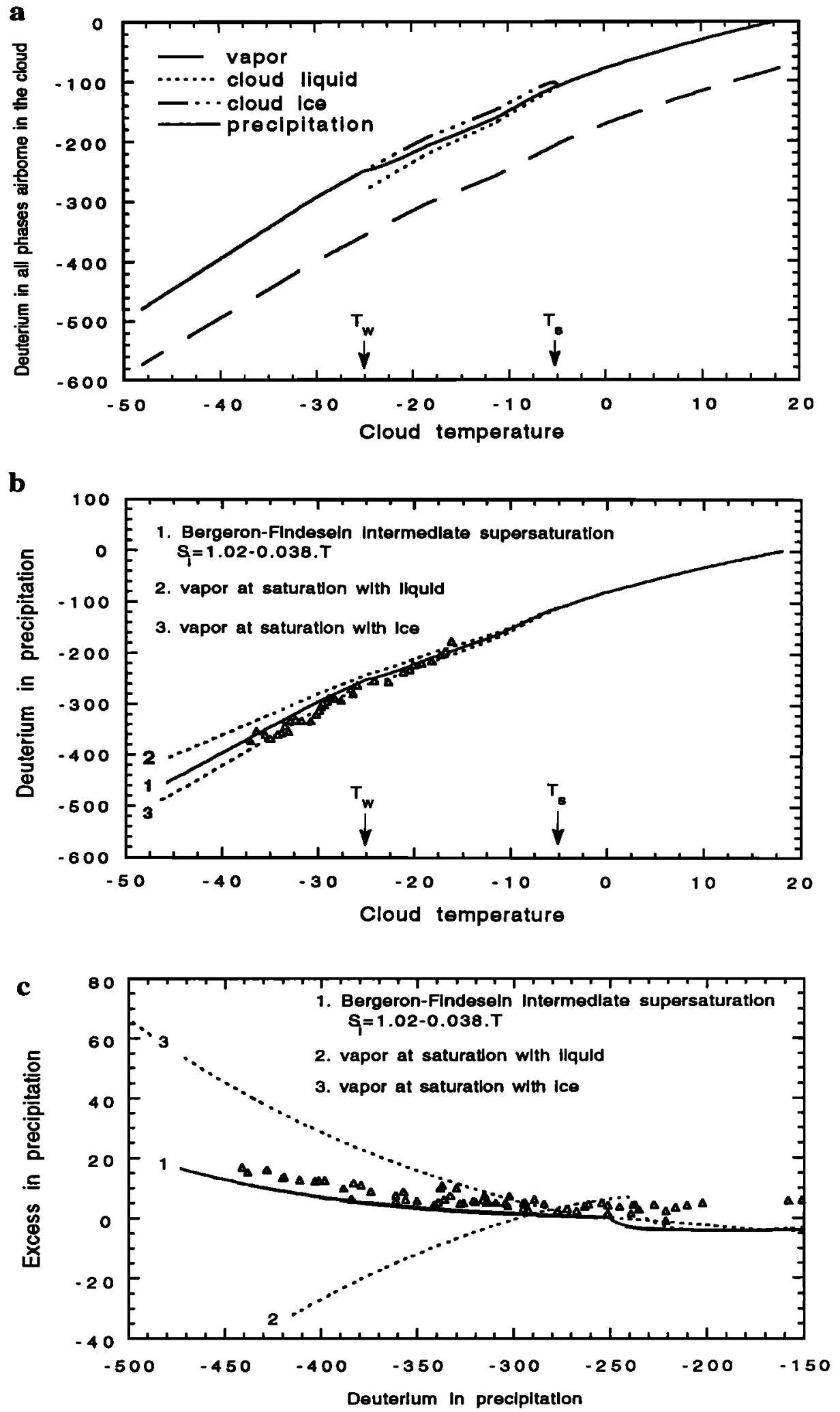


$$
\begin{gathered}
d m_{v}^{\prime}+d m_{l}^{\prime}+d m_{i}^{\prime}=-f_{i}^{\prime}-f_{i}^{\prime} \\
d m_{l}^{\prime}=-P_{l v}^{\prime}-P_{l i}^{\prime}-f_{i}^{\prime} \\
d m_{i}^{\prime}=P_{v i}^{\prime}+P_{l i}^{\prime}-f_{i}^{\prime}
\end{gathered}
$$

The mean isotopic composition of the total precipitation $(\bar{\delta})$ is a composite term which includes the precipitation derived from airborne liquid and from airborne ice. This is expressed by

$$
(1+\bar{\delta})=\frac{f_{i}^{\prime}+f_{i}^{\prime}}{f_{l}+f_{i}}
$$

The system of equations is solved by differentiating equations (19) to (21), combining them into the isotopic mass balance equation (24) to obtain the infinitesimal variation in $\delta$ of the vapor and in $\delta$ of the ice between two successive condensation steps. This leads to (26) and (27).

$$
\begin{aligned}
& \frac{d \delta_{v}}{\left(1+\delta_{v}\right)}=\frac{-1}{\left(m_{v}+\alpha_{k l} m_{l}\right)}\left(m_{l} d \alpha_{k l}-\right. P_{l v} \alpha_{k l} \\
&\left.+P_{v i} \alpha_{k i}+d m_{v}\right) \\
& d \delta_{i}=\frac{1}{m_{i}}\left[-\left(1+\delta_{i}\right)\left(P_{l i}+P_{v i}\right)+\left(1+\delta_{v}\right) \alpha_{k i} P_{v i}\right. \\
&\left.+\left(1+\delta_{v}\right) \alpha_{k l} P_{l i}\right]
\end{aligned}
$$

Equations (28) and (29), together with (17), determine completely the isotopic composition of each airborne phase in the cloud. The composition of the precipitation derives from (27). The results of the MCIM are discussed in section 3. The sensitivity of the model is discussed with respect to the supersaturation and to the specificities of mixed cloud processes. The influence of evaporative conditions at the mois-

Figure 6. (Opposite) (a) Deuterium content $(\delta D)$ in the different constituents of the cloud as a function of cloud temperature. The curves correspond to the standard run of the MCIM model where the supersaturation below $T=T_{w}$ is given by $S_{t}=1.02-0.0038 \times T$. The $\delta D$ of the vapor is lower by approximately $100 \%$ than $\delta D$ of any airborne condensed phase. The $\delta D$ of the liquid is lower than $\delta D$ of the ice, mostly because the cloud ice keeps an isotopic memory of ice formed at previous condensation steps. The $\delta D$ in precipitation lies in between $\delta D$ of solid and $\delta D$ of liquid as the precipitation removed from the system is derived from both condensed phases. (b) Deuterium in precipitation versus cloud temperature for different saturation conditions $\left(S_{i}\right)$ in the MCIM model. 1. BergeronFindesein hypothesis where $S_{i}$ takes intermediate values between saturation over ice and saturation over liquid. Curve 1 plots the standard run of the MCIM model $\left(S_{i}=\right.$ $1.02-0.0038 \times T)$. Curve 2: the vapor is at saturation with respect to cloud liquid. Curve 3: the vapor is at saturation with respect to cloud ice. Triangles are observations made by Lorius and Merlivat [1977] in East Antarctica (ground temperatures have been turned into cloud temperatures using $T_{\text {cloud }}=0.67 \times T_{\text {ground }}-1.2$ ). (c) Excess in precipitation versus $\delta D$ in precipitation for different saturation conditions $\left(S_{i}\right)$ in the MCIM model. Curves 1,2 , and 3 correspond to Figure 6a. Triangles are observations compiled by Petit et al. [1991]. ture source on the MCIM deuterium excess is discussed in section 4 .

\section{Results and Sensitivity Study of the MCIM Model}

\section{Results}

Mixing ratios. Figure 5 shows the mixing ratios of the vapor, the cloud liquid, and the cloud ice. The vapor is progressively converted into ice and its mixing ratio $m_{v}$ decreases during the air parcel history. The liquid mixing is equal to $m_{l 0}$ at the onset of the Bergeron-Findesein process $\left(T_{s}\right)$, but droplets are partly frozen and partly evaporated so that $m_{l}$ is equal to zero at $T_{w}$. Ice forms in the cloud starting at $T_{s}$ as a result of droplets freezing and vapor condensation. If no precipitation occurred, ice crystals would accumulate in the cloud and eventually represent the whole airborne condensed phase. This is not the case because the precipitation $\left(f_{i}\right)$ causes $m_{i}$ to decrease at low temperatures. At the lower end of the Bergeron-Findesein domain $\left(T_{w}\right)$, the amount of ice in the cloud is already so reduced that the system practically follows the conditions of a Rayleigh distillation.

Simulated $\delta s$. Figure 6a shows $\delta$ of all airborne phases in the MCIM, as a function of temperature. Because the fractionation pertaining to the vapor-liquid transition is lower than for the vapor-ice transition, the newly formed liquid has a lower $\delta$ than the newly formed ice. Moreover, the airborne ice sequestered in the cloud keeps a memory of previous condensation steps (with a higher $\delta$ ), whereas the liquid is fully equilibrated with the vapor at each step. This drives the $\delta$ value of airborne ice above the $\delta$ of airborne liquid (Figure 6a). Generally, $\delta$ in precipitation lies between $\delta$ of the liquid and $\delta$ of the ice. The MCIM $\delta$ in precipitation agrees well with observations in Antarctica, both at low temperatures (between $-20^{\circ} \mathrm{C}$ and $40^{\circ} \mathrm{C}$ ) and intermediate temperatures (between $0^{\circ} \mathrm{C}$ and $20^{\circ} \mathrm{C}$ ). Realistic, very negative, $\delta D$ values close to $-400 \%$ are obtained when the cloud temperature reaches $-40^{\circ} \mathrm{C}$. Such an agreement of onedimensional isotopic models with observations at low temperatures is not a specific performance of the MCIM (the model of Jouzel and Merlivat, for instance, is also quite satisfying from this point of view). However, for intermediate temperatures, the agreement with observations is slightly improved in the MCIM compared to previous models. This is because there is no discontinuity in $\delta$ at $T_{s}$ in the MCIM.

Sensitivity of the MCIM model to the saturation conditions of the vapor in the cloud. Figure $6 \mathrm{~b}$ displays $\delta D$ in precipitation versus temperature simulated by the MCIM for three different assumptions on the saturation conditions of the vapor. Specifically, we tested three possibilities for the vapor in the cloud (1) in between saturation with ice and saturation with respect to liquid (Bergeron-Findesein hypothesis), (2) saturated with respect to liquid, and (3) saturated with respect to ice. Between $T_{s}$ and $T_{w}$ the simulated $\delta D$ values do not differ by more than $20 \%$ o between extreme cases (2) and (3). For temperatures below $T_{w}$ this discrepancy increases up to $60 \%$. Figure $6 \mathrm{~b}$ suggests a better agreement of the simulated $\delta D$ with the observations when the supersaturation takes its values between cases 1 and 3 . Figure $6 \mathrm{c}$ shows the behavior of the deuterium excess in the MCIM for cases (1), (2), and (3). The $d$ values increase 
inland, as effectively observed in Antarctica, except when the vapor is kept at saturation with the liquid as in case (2). This suggests that the vapor is probably never at saturation with the liquid in polar clouds. Also, when the vapor is kept at saturation with the solid, the MCIM overestimates $d$ values $(d=+40$ to $+50 \%$ ). Figure $6 \mathrm{c}$ suggests that only an intermediate supersaturation analogous to case (1) yields a correct agreement with the data. We made several runs of the MCIM using various slopes for $S_{i}(T)$ as in the work of Fisher [1991]. These runs showed that $d$ in the MCIM is very sensitive to $S_{i}$. The sensitivity of the MCIM to the supersaturation was similar in magnitude, although slightly lower, than in the works of Jouzel and Merlivat [1984] or Fisher [1991]. For instance, an increase in $S_{i}$ from 1.2 to 1.5 at $-40^{\circ} \mathrm{C}$ has the effect to increase the value of $d$ in the MCIM by $+10 \%$. The best agreement with the observations was found in the supersaturation range 1.15 to 1.25 . Such a range appears plausible in view of the few existing observations [Miller and Schwerdtfeger, 1972; International Atomic Energy Agency (IAEA), 1969-1986].

\section{Sensitivity of the Model}

We have shown that the sensitivity of the MCIM to the supersaturation was similar to previous one-dimensional isotopic models. We now examine features that are specifcally new in the MCIM. First, droplets and ice crystals coexist between $T_{s}$ and $T_{w}$. Second, a nonzero amount of condensed phase is kept within the cloud. The first point is addressed by varying the nature (liquid or solid) of the airborne condensed phase. The second point is examined by testing different amounts of condensate in the cloud, which is equivalent in comparing the MCIM with the model of Jouzel and Merlivat (Rayleigh distillation).

Coexistence of liquid and ice. We define the nature of the airborne condensed phase as the relative proportion of liquid droplets versus ice crystals during the Bergeron-Findesein process. The variable $\rho$ is constructed as the ratio of the droplets to the total airborne condensed phase in between $\left(T_{s}, T_{w}\right)$. Provided that the precipitation intensity is maintained constant, $\rho$ depends only on the evaporation and on the freezing of the droplets (coefficients $C_{e}$ and $C_{f}$ ). In the real world, $\rho$ certainly differs among clouds and may possibly take any value between 0 and 1 . We limited ourselves to three test cases. We considered a typical mixed cloud ( $\rho=$ $0.5)$, a cloud made only with ice $(\rho=0)$, and a cloud made only with liquid $(\rho=1)$. In all three cases the total amount of condensed phase is the same.

The $\delta-T$ relations: Figure 7 a shows $\delta D$ versus $T$, respectively, for $\rho=0, \rho=0.5$, and $\rho=1$ (considering $\delta^{18} \mathrm{O}$ instead of $\delta D$ does not change the conclusions). The gradient $\delta D-T$ changes by no more than $1 \% \circ C^{-1}$ between extreme cases $\rho=0$ and $\rho=1$. This translates into a difference of $25 \%$ in $\delta D$ of precipitation at $-40^{\circ} \mathrm{C}$. The existing datasets of $\delta$ in surface snow do not allow to sort out this effect. Also, due to a less efficient fractionation for the liquid-vapor transition than for the vapor-ice transition, $\delta$ of the condensed phase decreases more rapidly with $T$ when the cloud contains a higher amount of liquid. Below $T_{w}$, we deal with ice crystals only and the system approaches a Rayleigh distillation. In this range of temperature the MCIM gives a $\delta D-T$ gradient of $6.5 \% C^{-1}$, a value close to Rayleightype models which is in fair agreement with the empirical gradient of $6.04 \% \mathrm{C}^{-1}$ proposed by Lorius and Merlivat
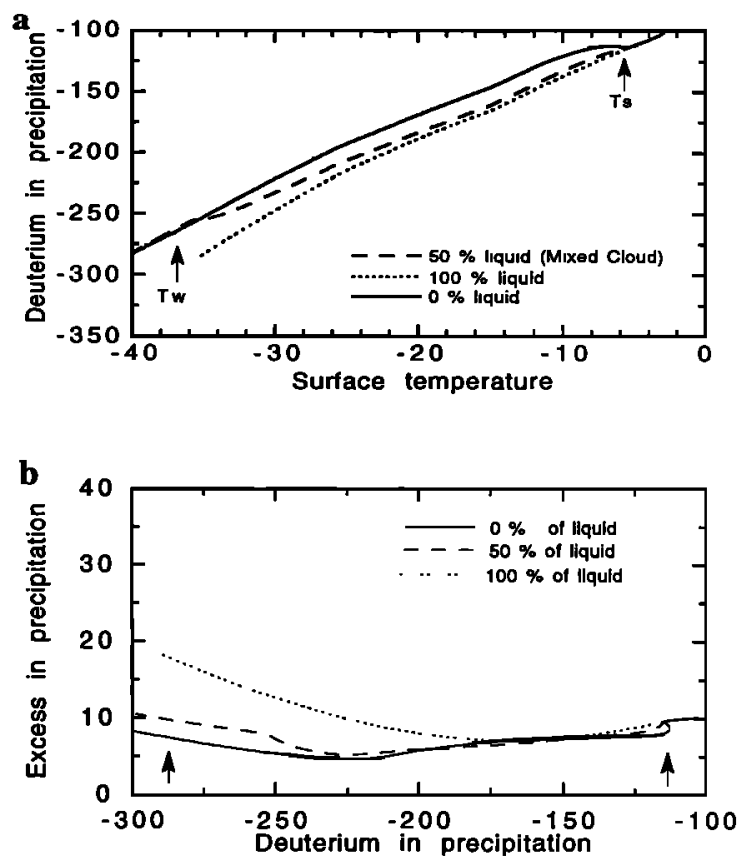

Figure 7. Sensitivity of the MCIM isotopic model to different compositions (liquid/solid) for the total airborne condensed phase (parameter $\rho$ in section 3 ). The total amount of the airborne phase is the same in all experiments. (a) $\delta D$ in precipitation versus temperature. (b) Excess in precipitation versus $\delta D$ in precipitation. Vertical arrows are boundaries of the Bergeron-Findesein domain $\left(T_{s}, T_{w}\right)$.

[1977] in East Antarctica. Our conclusion is that the nature of the condensed phase formed under the BergeronFindesein conditions does not strongly influence the $\delta-T$ relationship.

The $\delta D-\delta^{18} 0$ relationship: Although the gradients $\delta D-$ $\delta^{18} \mathrm{O}$ obtained in the MCIM for the three cases of $\rho$ were all fairly similar (not shown), $d$ proved to be more sensitive (Figure 7b). It is observed that between cases $\rho=0$ and $\rho=$ $1, d$ differs by several per mils. However, an "all liquid" configuration ( $\rho=1)$ must be improbable, considering that supercooled droplets are far from the equilibrium with the vapor. Besides, $d$ obtained in the model for a mixed cloud ( $\rho$ $=0.5)$ and for an "all ice" cloud $(\rho=0)$, was not very different, even at low temperatures.

Amount of condensed phase kept in the cloud. We examine now the consequences of keeping in the cloud various quantities of a condensed phase during the BergeronFindesein process. In the MCIM the total airborne condensed phase is entirely controlled by the precipitation intensity. A systematic removal of the condensed phase formed at each condensation represents the extreme case of Rayleigh distillation conditions, whereas no removal would correspond to a hypothetical closed system [Jouzel, 1986]. We constructed the variable $\omega$ in such a manner that $\omega=0$ in a Rayleigh distillation and $\omega=1$ for a closed system where all the condensed phase is kept in the cloud. By setting the values of $f_{i}$ and $f_{l}, \omega$ can be varied within the interval $[0,1]$.

The $\delta-T$ relations: The gradients $\delta D-T$ obtained for various amounts of condensed phase are shown in Figure 8a. It is clear that the gradient $\delta D-T$ is sensitive to $\omega$ (a higher $\omega$ drives a smaller gradient $\delta D-T$ ). This can be 
understood as a "memory" of the airborne phase formed at previous steps which progressively accumulates into the cloud. If more airborne phase is kept ( $\omega$ is higher), the precipitation has a higher $\delta$. Within the interval $\left(T_{s}, T_{w}\right)$ the gradient $\delta D-T$ changes by a factor 3 when passing from Rayleigh to closed system conditions. However, a closed system cloud type is only approached in the inner core of very strong convective clouds, and the most frequent values of water content found in all types of stratiform clouds are within the narrow range of $0.05-0.25 \mathrm{gm}^{-3}$, generally less for stratiform clouds in polar regions [Mason, 1971]. As an example, Miller [1973] infers from crystal growth calculations that a crystal mass density of $5.10^{-9} \mathrm{gm}^{-3}$ can be expected at the top of the inversion layer over the Antarctic plateau. Multiplying this estimate by a factor as large as $\mathbf{1 0 0 0}$ would still leave $\omega$ very low. In Antarctica as well as in Greenland [Johnsen et al., 1989], $\delta$ measured in snow is consistent only with low values of $\omega$ in isotopic models. This may explain the overall good performances of previously used Rayleigh-type isotopic models, despite their simplistic characteristics.

The $\delta D-\delta^{18} \mathrm{O}$ relationships: The gradients $\delta D-\delta^{18} \mathrm{O}$ simulated by the MCIM for different values of $\omega$ do not deviate significantly from the meteoric water line, which suggests an observational gradient $\delta D-\delta^{18} \mathrm{O}$ close to 8 [Craig and Gordon, 1965]. Regarding the deuterium excess, as long as $\omega$ does not exceed 10 to $15 \%$, the MCIM is able to match correctly the observations, including the deuterium excess of inland Antarctica that can be as large as $+20 \%$ o [Petit et al., 1991]. Again, a reasonable agreement with the $d$
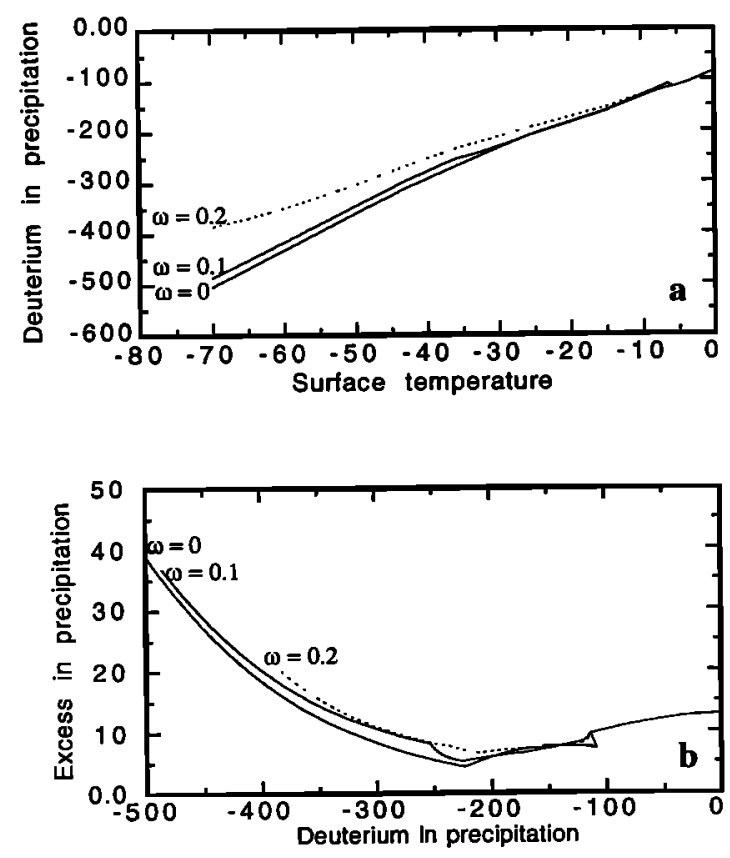

Figure 8. Sensitivity of the MCIM isotopic model to different amounts of total airborne condensed phase (total is equal to liquid plus ice). The amount of total condensed phase is varied by changing the value of $\omega$ in section 3 ( $\omega=$ 0 means Rayleigh distillation, $\omega=1$ means closed system without precipitation). (a) $\delta D$ in precipitation versus temperature. (b) Excess in precipitation versus $\delta D$ in precipitation.

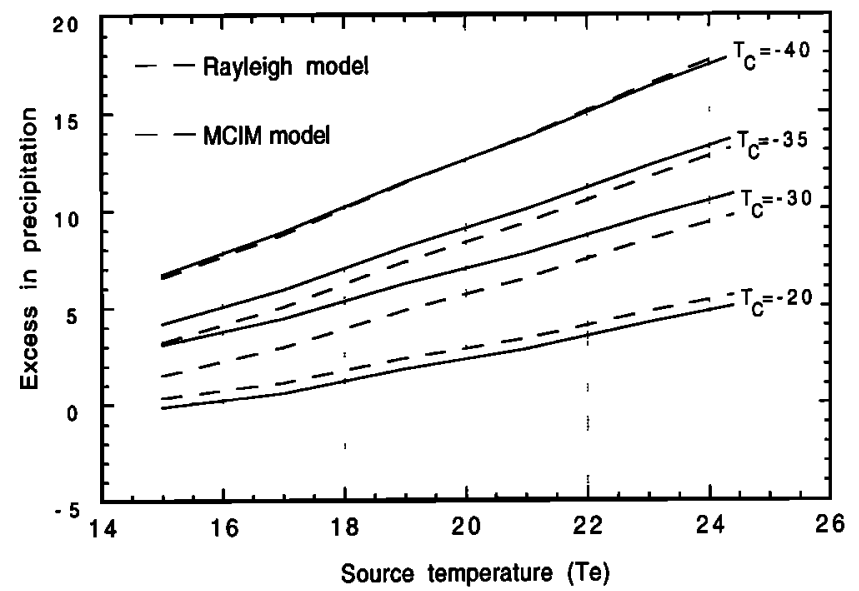

Figure 9. Response of $d$ in precipitation to the evaporative source temperature $\left(T_{e}\right)$. The response is plotted at different condensation temperatures going from $T_{C}=-20^{\circ} \mathrm{C}$ to $T_{C}=$ $-40^{\circ} \mathrm{C}$. Note that $d$ is more sensitive to $T_{e}$ when going inland. The results obtained using the MCIM isotopic model (solid curves) are compared to those obtained using the RMK model of Jouzel and Merlivat (dashed curves).

observations can be reached only when the system is close to the Rayleigh conditions at low temperature.

\section{Influence of the Evaporative Source Conditions on $d$ Values in Precipitation}

Previous works have outlined the fact that $d$ in polar precipitation is largely determined by the source conditions, specifically by the following three parameters: sea surface temperature $\left(T_{e}\right)$, relative humidity $(H)$, and wind speed $(W)$. This has motivated attempts to use $d$ as a proxy of ocean surface conditions [Jouzel et al., 1982; Johnsen et al., 1989; Petit et al., 1991]. In this section we examine the sensitivity of the MCIM model to the source conditions and compare it with previous one-dimensional isotopic models (RMK model).

Sea surface temperatures. Petit et al. [1991] pointed out that the sea surface temperature (SST) largely determines $d$ values in Antarctic snow. They also demonstrated that warmer SST result in higher $d$ in snow, especially at inland sites (mean surface temperatures lower than $-30^{\circ} \mathrm{C}$ ). The question is how the implementation of the BergeronFindesein process modifies these conclusions. For both the MCIM and the RMK models, Figure 9 shows $d$ versus $T_{e}$ at different in situ condensation temperatures. Globally, the sensitivity of the MCIM model to $T_{e}$ is similar to that of the RMK. Whatever the in situ temperature the maximum discrepancy between both models indeed does not exceed $2 \%$ for the excess. Using the partial derivative $\sigma_{t}=\partial d / \partial T_{e}$, which measures the response of $d$ in precipitation to changes in SST, we found that $T_{e}$ is about 3 times more important in governing the excess in snow at $-40^{\circ} \mathrm{C}$ than at $-20^{\circ} \mathrm{C}$. The difference in $\sigma_{t}$ obtained in the RMK and MCIM models, respectively, at a given temperature is small.

Relative humidity and wind speed. In agreement with Petit et al., the MCIM model produces lower deuterium excesses in response to increased source humidity. The sensitivity of $\boldsymbol{d}$ to changes in $\boldsymbol{H}$ being assessed by the value 
of $\sigma H=\partial d / \partial H$ at a given condensation temperature, we found that $\sigma H$ in the MCIM decreases by a factor 2 between coastal $\left(-20^{\circ} \mathrm{C}\right)$ and inland locations $\left(-40^{\circ} \mathrm{C}\right)$. In other terms the memory kept by $d$ of changes in $H$ at the source is larger on the coast than over the central ice sheet regions. When it comes to the comparison of the MCIM to the RMK model, the response of $d$ to $H$ is very similar. The wind speed above the ocean surface triggers the initial fractionation coefficient, an effect which is expected to be preserved through the cooling history and seen in $d$ of precipitation. However, the excess is not a monotone function of the wind speed, so that low and high wind speed values can yield identical $d$ values [see Petit et al., 1991]. In addition, the high-frequency variability of the wind is likely to smooth out its influence on the deuterium excess. We will not discuss in detail this problem. In summary, regarding the response of $d$ to the source conditions, the results of the MCIM model fully endorse the conclusions of the detailed sensitivity study presented by Petit et al. [1991].

\section{Conclusion}

The main difference between the MCIM isotopic model presented in this study and the previous one-dimensional isotopic models consists in a more refined description of the microphysical processes occurring at low temperatures. Below $0^{\circ} \mathrm{C}$ and down to $-15^{\circ}$ to $-30^{\circ} \mathrm{C}$, we allow a certain fraction of the preexisting liquid, formed at higher temperatures, to remain in the cloud. Simultaneously, the solid phase that progressively appears is not systematically removed. In this case, the water vapor mixing ratio takes an intermediate value between saturation over ice and saturation over water. Droplets evaporate, whereas the vapor tends to condense on the growing ice crystals. The predicted $\delta-T$ gradients and $d$ in precipitation compare well with the available data in Antarctic surface snow, but they are not very sensitive to the quantity of liquid or solid kept in the cloud. On the other hand, in accordance with previous models, $d$ in the MCIM remains strongly dependent on changes in the supersaturation function. The impact of climatic changes at the evaporative moisture source on the $d$ values shows quite similar features than those previously obtained using Rayleightype models. In summary, taking into account realistically the processes that lead to the formation of the precipitation does not imply reassessing the climatic information previously derived from the deuterium excess.

Nevertheless, the present study concerns a given air parcel and does not simulate the conditions of the wide scale poleward moisture transport. In particular, we did not discuss the nature of the trajectory, nor the eventual mixing of the air during the isotopic history. The fact that the $\delta$ in snow, at a given location, results from several precipitation events, possibly characterized by different origins [Fisher, 1990], has also not been envisaged. Simulations of the water isotope cycle using GCMs [Joussaume et al., 1984; Jouzel et al., 1987] seems a promising approach for solving these problems as their results may help to constrain the moisture transport of the present one-dimensional model. In addition, GCMs make it possible to take into consideration the geographical variability of $\delta$ in the original oceanic vapor, which plays a key role in governing the $d$ values.

\section{Appendix: Pseudoequilibrium Fractionation Coefficient for Liquid-Vapor Phase Transition out of Saturation}

We infer the pseudoequilibrium fractionation factor $\alpha_{k l}$ from the isotopic evolution of a liquid droplet growing from the surrounding vapor as a result of molecular collisions. Growth by coalescence has not been taken into account because its contribution becomes important only if the droplet radius exceeds $30 \mu \mathrm{m}$. The saturation with respect to the liquid-vapor equilibrium is expressed by $S_{l}$, defined by

$$
S_{l}=\frac{e_{w}}{e_{l}^{s}}
$$

Values of $S_{l}$ lower than 1 account for liquid droplets placed in undersaturated vapor, which corresponds specifically to the conditions of the Bergeron-Findesein process and has for consequence the progressive disparition of the droplets by evaporation. The rate of change in the isotopic ratio of a droplet in such an environment is expressed by (A2) [Stewart, 1975]:

$$
\begin{aligned}
\frac{d R_{L}}{d t}=\frac{3 \rho_{\text {sat }}}{a^{2} \rho_{\text {drop }}} & {\left[D ^ { \prime } f ^ { \prime } \left[S_{L}\left(R_{v}-\frac{R_{L}}{\alpha_{L}}\right)\right.\right.} \\
& \left.\left.+\frac{A}{\alpha_{L}}\left(R_{L}\right)\left(S_{L}-1\right)\right]-A D f\left(R_{L}\right)\left(S_{L}-1\right)\right]
\end{aligned}
$$

where

$a$ droplet average radius;

$\rho_{\text {drop }}$ droplet average density;

$\rho_{\text {sat }}$ vapor density at saturation;

$D$ diffusivity of water vapor in the air;

$f$ ventilation coefficient of the water vapor [Kinzer and Gunn, 1951, p. 74];

$D^{\prime}, f^{\prime}$ same for isotopic species;

$A$ droplet vapor heat transfer coefficient [Jouzel, 1974, p. 38];

$\alpha_{L}$ equilibrium fractionation coefficient [Majoube, 1971].

Equation (A2) depends on the isotope ratios of the droplets and the surrounding vapor, $\boldsymbol{R}_{L}$ and $\boldsymbol{R}_{v}$, respectively, in such a manner that it can be rewritten under the form

$$
\frac{d R_{L}}{d t}=a_{1} R_{L}+a_{2} R_{v}
$$

The expression of the factors $a_{1}$ and $a_{2}$ is given by

$$
\begin{aligned}
a_{1}= & \frac{3 \rho_{\text {sat }}}{a^{2} \rho_{\text {drop }}}\left[-\frac{D^{\prime} f^{\prime}}{\alpha_{L}}\left(S_{L}+\frac{A D^{\prime} f^{\prime}}{\alpha_{L}}\right)\right. \\
& \left.\cdot\left(S_{L}-1\right)-A D f\left(S_{L}-1\right)\right] \\
a_{2}= & \frac{3 \rho_{\text {sat }}}{a^{2} \rho_{\text {drop }}}\left(D^{\prime} f^{\prime}\right) S_{L}
\end{aligned}
$$

Assuming a constant isotopic ratio for the vapor $\left(R_{\nu}\right),(\mathrm{A} 2)$ has the solution

$$
a_{1} R_{L}+a_{2} R_{v}=\left(a_{1} R_{L}+a_{2} R_{v}\right)_{t=0} e^{a_{1} t}
$$


The term $\tau=\left(a_{1}\right)^{-1}$ is the $e$-folding isotopic relaxation time of the droplet. The range of possible values for $\tau$ in cloud droplets has been studied by Jouzel [1974], who reached the conclusion that $\tau$ does not exceed a few seconds as long as the droplet radius remains smaller than $30 \mu \mathrm{m}$. In the case of larger drops found in convective clouds, $\tau$ would be significantly higher and the drop would no longer be in isotopic equilibrium. As we address here processes occurring in a stratiform cloud, it is reasonable to postulate that $t \gg \tau$. Hence the droplets are isotopically equilibrated, although they are not saturated with respect to the vapor (we call that "pseudoequilibrium" in section 3). Therefore the right member of (A4) is equal to zero, which yields the expression of the pseudoequilibrium fractionation factor $\alpha_{k l}$ :

$$
\begin{aligned}
\alpha_{k l}=\frac{R_{l}}{R_{\nu}}=S_{l}\left(D^{\prime} f^{\prime}\right)\left[S _ { l } \left(\frac{D^{\prime} f^{\prime}}{\alpha_{l}}-\right.\right. & \left.\frac{A D^{\prime} f^{\prime}}{\alpha_{l}}+A D f\right) \\
& \left.+\frac{A D^{\prime} f^{\prime}}{\alpha_{l}}-A D f\right]^{-1}
\end{aligned}
$$

One can note that $\alpha_{k l}$ depends both on the equilibrium fractionation factor $\alpha_{l}$ and on $S_{l}$ which characterizes the departure from saturation conditions with respect to the liquid-vapor transition. In the limit case where the population of droplets is in thermodynamic equilibrium $\left(S_{l}=1\right)$, (A5) simplifies into $\alpha_{k l}=\alpha_{l}$.

\section{References}

Bergeron, T., On the physics of clouds and precipitation, Proc. 5th Assemb. UGGI Lisbon, 2, 156, 1935.

Bigg, E. K., The supercooling of water, Proc. Phys. Soc. London B, $66,688-694,1953$.

Craig, H., and L. I. Gordon, Deuterium and oxygen 18 variation in the ocean and marine atmosphere, in Stable Isotopes in Oceanographic Studies and Paleotemperatures, edited by E. Tongiorgi, pp. 9-130, Spoleto, Italy, 1965.

Dansgaard, W., Stable isotopes in precipitation, Tellus, 16, 436-468, 1964.

Dansgaard, W., J. W. C. White, and S. J. Johnsen, The abrupt termination of the Younger Dryas, Nature, 339, 532-534, 1989.

Federer, B., N. Brichet, and J. Jouzel, Stable isotopes in hailstones, 1, The isotopic cloud model, J. Atmos. Sci., 39(6), 1323-1336, 1982.

Fisher, D. A., A zonally averaged stable-isotope model coupled to a regional variable elevation stable isotope model, Ann. Glaciol., 14, 65-72, 1990.

Fisher, D. A., Remarks on the deuterium excess in precipitation in cold regions, Tellus, 43(B), 401-407, 1991.

Fisher, D. A., Possible ice-core evidence for a fresh melt water cap over the Atlantic Ocean in the Early Holocene, in The Last Deglaciation: Absolute and Radiocarbon Chronologies, edited by E. Bard and W. S. Broecker, Springer-Verlag, New York, 1992.

Fisher, D. A., and B. T. Alt, A global oxygen isotope modelSemiempirical, zonally averaged, Ann. Glaciol., 7, 117-124, 1985.

Friedman, I., A. C. Redfield, B. Schoen, and J. Harris, The variations of the deuterium content of natural waters in the hydrologic cycle, Rev. Geophys., 2, 177-224, 1964.

International Atomic Energy Agency (IAEA), Environmental isotope data $n^{\circ} 1-8$, World survey of isotope concentration in precipitation, Tech. Rep. Ser. 96, 117, 129, 147, 165, 192, 226, 264, Vienna, 1969-1986.

Johnsen, S. J., W. Dansgaard, and J. W. C. White, The origin of Arctic precipitations under present and glacial conditions, Tellus, 4l(B), 452-468, 1989.

Joussaume, S., J. Jouzel, and R. Sadourny, Water isotope cycles in the atmosphere, First simulation using a general circulation model, Nature, 311, 24-29, 1984.

Jouzel, J., Complémentarité des mesures de deutérium et de tritium pour l'étude de la formation des grélons, exemple d'application, Ph.D. thesis, 160 pp., Univ. de Paris Sud, Centre d'Orsay, 1974.

Jouzel, J., Isotopes in cloud physics: Multiphase and multistage condensation processes, in Handbook of Environmental Isotopes Geochemistry, vol. 2, pp. 63-111, Elsevier, New York, 1986.

Jouzel, J., and L. Merlivat, Deuterium and oxygen 18 in precipitation: Modeling of the isotopic effects during snow formation, $J$. Geophys. Res., 89, 11,749-11,757, 1984.

Jouzel, J., L. Merlivat, and E. Roth, Isotopic study of hail, $J$. Geophys. Res., 80, 5115-5030, 1975.

Jouzel, J., N. Brichet, B. Thalman, and B. Federer, A numerical cloud model to interpret the isotope content of hailstones, Com. Conf. Int. Phys. Nuages, 8, 249-252, 1980.

Jouzel, J., L. Merlivat, and C. Lorius, Deuterium excess in an East Antarctic ice core suggests higher humidity at the ocean surface during the last glacial maximum, Nature, 5885, 688-691, 1982.

Jouzel, J., G. L. Russell, R. J. Suozzo, R. D. Koster, J. W. C. White, and W. S. Broecker, Simulations of the HDO and $\mathrm{H}_{2}{ }^{18} \mathrm{O}$ atmospheric cycles using the NASA/GISS general circulation model: The seasonal cycle for present-day conditions, J. Geophys. Res., 92, 14,739-14,760, 1987.

Kinzer, G. D., and R. Gunn, The evaporation, temperature and thermal relaxation-time of freely falling waterdrops, $J$. Meteorol., 8(2), 71-83, 1951.

Lorius, C., and L. Merlivat, Distribution of mean surface stable isotope values in East Antarctica: Observed changes with depth in a coastal area, in Isotopes and Impurities in Snow and Ice, Publ. 118 , pp. 125-137, Int. Assoc. Hydrol. Sci., Gentbrugge, Belgium, 1977.

Majoube, M., Fractionement en oxygène 18 et en deutérium entre l'eau et sa vapeur, J. Chim. Phys., 10, 1473, 1971.

Mason, B. J., The Physics of Clouds, 671 pp., 2nd ed., Clarendon, Oxford, 1971.

Merlivat, L., and Jouzel, J., Global climatic interpretation of the deuterium-oxygen 18 relationship for precipitation, $J$. Geophys. Res., 84(C8), 5029-5033, 1979.

Miller, S., An analysis of heat and moisture budgets of the inversion-layer for steady-state conditions over the Antarctic Plateau, Ph.D. thesis, Univ. Wisconsin, 94 pp., 1973.

Miller, S., and W. Schwerdtfeger, Ice crystal formation and growth in the warm layer above the Antarctic temperature inversion, Antarctic J. U.S., 7(7), 170-171, 1972.

Petit, J. R., J. W. C. White, N. W. Young, J. Jouzel, and Y. S. Korotkevitch, Deuterium excess in recent Antarctic snow, $J$. Geophys. Res., 96(D3), 5113-5122, 1991.

Pruppacher, H. R., and J. D. Klett, Microphysics of Clouds and Precipitations, 713 pp., D. Reidel, Norwell, Mass., 1978.

Rogers, R. R., A Short Course in Clouds Physics, vol. 96, 235 pp., Pergamon, New York, 1979.

Siegenthaler, $U$., and $H$. Matter, Dependence of $d^{18} O$ and $d D$ in precipitation on climate, in Paleoclimate and Paleowaters: $A$ Collection of Environmental Isotope Studies, pp. 37-51, International Atomic Energy Agency, Vienna, 1983.

Stewart, M. K., Stable isotope fractionation due to evaporation and isotopic exchange of falling water drops: Application to atmospheric processes and evaporation of lakes, J. Geophys. Res., 80, 1133-1146, 1975.

Taylor, C. B., The vertical variations of the isotopic concentrations of tropospheric water vapour over continental Europe and their relationship to tropospheric structure, N. Z. Dep. Sci. Ind. Res., Inst. Nucl. Sci. Rep., INS-R-107, 45 pp., 1972.

P. Ciais, Stable Isotope Laboratory, University of Colorado INSTAAR, Campus Box 450, Boulder, CO 80303.

J. Jouzel, Laboratoire de Glaciologie et de Géophysique de l'Environnement, BP 96, 38402, St. Martin d'Hères, France.

(Received February 19, 1993; revised February 7, 1994; accepted February 8, 1994.) 